\title{
Recent Updates on Essential Tremor
}

\author{
Fariha Jamal, MD*
}

Department of Neurology, School of Medicine, Baylor College of Medicine, Texas, USA

*Corresponding author: Fariha Jamal, MD, Assistant Professor, Department of Neurology, School of Medicine, Baylor College of Medicine, Texas, USA

\begin{abstract}
Essential Tremor is the most common movement disorder, but many aspects of this common condition are unclear. There have been multiple updates in the field of Essential Tremor in last few years, that has improved our understanding to some extent. Recently, consensus criteria for classifying tremor disorders was published. Special emphasis was on Essential Tremor, to facilitate better phenotyping and understanding of underlying etiology. Purpose was to identify homogenous population of different tremor syndromes. In addition, recent work focused on developing non-motor neuropsychological tests that can be used for Essential Tremor patients. A study used these tests to identify $\mathrm{MCl}$ subtypes associated with Essential Tremor. Further studies have been conducted to study gait and mood difficulties in Essential Tremor patients. Concept of early and late onset Essential Tremor as distinct clinical entities was not endorsed by recent neuropathological study. MRI guided focused ultrasound thalamotomy was FDA approved for patients with refractory Essential Tremor. This review summarizes the recent advances in the field of Essential Tremor.
\end{abstract}

\section{Keywords}

Essential Tremor, Classification, Neuropathology, Cognition, Mortality, Treatment

Essential Tremor (ET) is the most common movement disorder [1]. Despite its common presence, exact etiology, phenotypic diversity, associated symptoms, clinical course, response to medications and surgery is unclear. The term "Essential Tremor" is widely used in neurology and at times diverse types of tremor are labelled as ET without clear understanding [2]. Due to lack of understanding, there are concerns for over-diagnosis and possibility of mismanagement. There have been attempts to develop a classification scheme for tremor to identify different phenotypes, underlying etiology and associated neurologic symptoms, but there are still dif- ferent opinions and lack of understanding among clinicians.

\section{Classification}

Initial consensus criteria for tremor classification was developed in 1998 by Movement Disorder Society [3]. It was recognized that different controversies exist, and similar phenotypes can be classified differently based on individual observation [4]. Diagnosis of tremor was based on clinical classification only, as etiologies are unclear and multifactorial. Clinical diagnosis was based on history and examination.

Meaning of Essential Tremor has evolved over time [5]. For a long time, it was considered as a "pure tremor" with no other neurologic and non-neurologic symptoms and signs [6]. Presence of other neurologic signs virtually excluded ET as a possible diagnosis, but this concept is changing rapidly. ET is now considered to be a "syndrome" that can be associated with other symptoms [7]. Consensus statement on the classification of tremor was revised and published by the task force on tremor of the International Parkinson and Movement Disorder Society in 2017 [8]. Task force defined tremor as an involuntary, rhythmic, oscillatory movement of a body part.

The proposed classification has two main axes. Axis 1 is based on clinical features and Axis 2 is based on etiology. Axis 1 is quite broad and includes clinical history, examination, laboratory tests, receptor imaging, and biomarkers. Clinical features to be considered in axis 1 include age of onset, family history, temporal evolution, exposure to drugs, toxins, body distribution, activation conditions, frequency and associated signs. Onset after 60 years of age is to be considered as late adulthood.

Citation: Jamal F (2019) Recent Updates on Essential Tremor. Int J Neurol Neurother 6:081. doi. org/10.23937/2378-3001/1410081

Accepted: May 08, 2019: Published: May 10, 2019

Copyright: (c) 2019 Jamal F. This is an open-access article distributed under the terms of the Creative Commons Attribution License, which permits unrestricted use, distribution, and reproduction in any medium, provided the original author and source are credited. 
No change was proposed in activation conditions. Two main activation conditions are rest and action tremor. Action tremor includes postural, simple kinetic, intentional, task specific and isometric tremor. Based on Axis 1, ET can be classified as Essential Tremor and Essential Tremor plus. Essential tremor is defined as bilateral upper extremity action tremor for at least 3-years duration, with or without tremor in other locations and no other neurological signs. ET plus is tremor with similar characteristics but may have additional neurological signs such as impaired tandem gait, memory, dystonia or other mild neurologic signs of unknown significance. Isolated voice, head, orthostatic, task and position specific tremor are not part of ET syndrome as per new classification scheme. 3-years duration was included to exclude the possibility of tremor syndrome evolving into another neurodegenerative syndrome.

\section{Etiology}

One of the goals of developing this classification system is to help with determination of underlying etiologies. For a while, it was considered to be a genetic disorder only. But due to phenotypic diversity, it is likely that underlying etiology is multifactorial and complex. Not a single gene has been linked to ET with certainty. Few genetic loci had been implicated e.g. 3q13, 2p22, $6 \mathrm{p} 23$ and other variants as per genome wide association studies but none had been accepted as the final genetic variable involved in pathogenesis of ET [9-11]. Besides, disease penetrance is incomplete, even in older age group [12]. Like other neurodegenerative disorders, there is a possibility that environmental exposure along with genetic predisposition triggers the pathway leading to clinical syndrome. Like genetic etiology, no single environmental toxin or exposure had been linked with ET pathogenesis. But, few studies have shown that toxins impacting cerebellum may be associated with increased risk of developing ET. These toxins include harmine, harmane, lead and ethanol abuse [13-15].

\section{Neuropathology}

There have been diverse opinions about the neuropathology underlying the genesis of ET. It was postulated for a long time that a central oscillator exists and pathology in such oscillator causes ET, though definite evidence to prove this had been lacking. Recently, postmortem studies in ET patients have demonstrated degenerative changes in cerebellum. Purkinje cells in cerebellum have demonstrated signs of injury. Torpedoes (swelling in Purkinje cell axons due to abnormal accumulation of proteinaceous material) were reported in injured Purkinje cells $[16,17]$. Abnormalities in the synaptic connections between cerebellar input fibers and Purkinje cell dendrites have been noted. Patients with ET had decrease in climbing fibers and Purkinje cell synaptic density [18]. Increase in synapses were noted with peripheral thin Purkinje cell spiny projections. All these changes are indicative of neurodegeneration in cerebel- lum. Louis, et al. demonstrated increase in number of cerebellar torpedoes in ET patients. In their study, morphological analysis of the Purkinje cells showed increase in dendritic swellings, axonal branching, axonal recurrent collaterals and terminal sprouting in ET patients as compared to healthy controls.

On the contrary, another postmortem series demonstrated Lewy bodies in ET brains. These bodies were abundant in locus ceruleus as compared to other brain stem nuclei $[19,20]$. Nor-epinephrine is the main neurotransmitter in locus ceruleus (LC). Purkinje cells receive projections from LC. Another postmortem study showed intranuclear inclusions in cerebellum [21]. These findings point toward cerebellum as the potential site of neurodegeneration in ET patients. Hence, within cerebellum, there are heterogenous pathological findings which may explain clinical heterogeneity.

\section{Age of Onset}

Another controversy that has arisen in recent years is to distinguish between ET cases based on age of onset. Deuschl, et al. proposed in 2014 that tremors occurring later in life (> 65 years) are not classical ET, rather an age-related tremor (ART). Incidence of ET increases with age. A meta-analysis suggested that action tremor may be seen in $10 \%$ of individuals above age 90 . They argued that ET is fully penetrant after age 65 years, hence, these patients have ART, a different disease entity as compared to classical ET. Their study suggested increasingly higher prevalence of ART with older age, faster progression and association with higher mortality [22]. A similar study suggested distinction between early and late-onset tremor [23]. At the same time, Louis, et al. have shown that penetrance of ET is not complete by the age of 65 years [12]. Hence, there is a possibility that age of onset and clinical progression is heterogeneous due to diverse etiology and pathogenesis. In another study, Kuo, et al. studied the postmortem changes in cerebellum of ET brains and included young and old onset. 30 ET cases with age of tremor onset $<50$ years, 30 ET cases with age of onset $>50$ years and 30 controls were included in the study. They also used alternative age of onset cut-points, 40 and 60 years. Similar pathologic findings were reported for early onset and late onset ET cases, and results stayed the same with all age of onset cut-points. Hence, this study did not support the earlier hypothesis of ART being a distinct clinic-pathological entity [24]. This is still an ongoing debate and needs to be proven in larger, prospective studies.

\section{ET and Cognition}

There is a growing notion that ET may be associated with cognitive deficits. Multiple cross-sectional studies have shown that ET patients may have deficits in executive functioning, attention, concentration, verbal fluency, naming, recent and working memory [25-29]. These deficits are due to alternations in circuitry between cer- 
ebellum, thalamus and cortex [30]. Recently, this work was expanded to develop motor free neuropsychological tools. Cersonsky, et al. identified five non-motor tests in the domains of memory and executive function that best predicted mild cognitive impairment in ET subjects. Tests included California Verbal Learning Test II Total Recall, Logical Memory II, Verbal-Paired Associates I, Category Switching Fluency and Color-Word Inhibition [31].

Mild cognitive impairment had been reported in ET patients. $\mathrm{MCl}$ subtypes in ET patients was explored by Park, et al. They found that most of ET patients (56\%) had non-amnestic $\mathrm{MCl}$ as opposed to amnestic $\mathrm{MCl}$ [32]. Collins, et al. conducted a study recently, which utilized motor-free neuropsychological tests. $71 \%$ of ET patients had normal cognition, $18.8 \%$ had $\mathrm{MCl}$ and $10 \%$ were reported to have dementia. $\mathrm{MCl}$ cases were further stratified into $\mathrm{MCl}$ subtypes. Majority of the cases were amnestic- $\mathrm{MCl}(70.8 \%)$ and majority of cases were also multi-domain $\mathrm{MCl}(66.7 \%)$. The most commonly affected domain was memory, followed by attention, executive function, language and then visuospatial. These results contrast with those reported by Park, et al. and previous studies in which executive dysfunction was the most common cognitive deficit [33]. These results need to be tested in larger, longitudinal studies, as these findings can have long term implications. Amnestic $\mathrm{MCl}$ in $\mathrm{ET}$ patients may be a marker of early neurodegenerative disorder, Alzheimer dementia or similar pathology, hence changing the approach how these patients will be followed and treated.

\section{ET and Gait}

There are multiple studies about association of ET and gait impairment, over the last two decades. Most of the studies used bedside clinical tests for gait examination. More mis-steps during tandem gait, reduced speed, impaired balance and slower walk were the common findings [34-37]. Some of the studies used quantitative gait assessment tools and reported comparable results [38]. Deficits were severe with increasing age and presence of head tremor. In a recent study, Louis, et al. compared gait and balance of first degree relatives of ET cases to that of age-matched controls. First degree relatives of ET patients reported more near-falls and reduction in balance confidence as compared to controls. They also had more difficulty in maintaining tandem stance. They concluded that this could represent a preclinical phase for ET, similar to seen in other neurodegenerative disorders [39].

\section{ET and Depression}

Depression has been studied in ET patients. Significantly increased depression had been reported in ET patients as compared to controls $[40,41]$. There have been conflicting reports of correlation between tremor severity and depression. Some studies found no correlation while others reported that depression was associated with worsening tremor severity $[27,29,42]$. Recently, Aslam, et al. compared 104 ET subjects with 481 non-ET controls. Hamilton Depression Scale, Geriatric Depression Scale and Neuropsychiatric Inventory were used for assessment. No significant differences in depression were found between ET and non-ET groups. In addition, there was no correlation between depression and tremor severity, duration or age of onset [43]. These results contrast with prior reports and merit further studies and continued assessment of ET patients for neuropsychiatric symptoms.

\section{Essential Tremor and Electrophysiology}

Use of electrophysiology to define different characteristics of tremor and distinguish between tremor syndromes has been explored in the past. None of the identified parameters were singled out as significant tool to discriminate between tremor types. In 2011, Nistico, et al. recruited 15 Parkinson disease (PD) and 15 ET patients for electrophysiological examination. They demonstrated that muscle burst duration and frequency was higher in ET patients as compared to PD. In addition, rest tremor in ET patients showed a synchronous pattern where PD patients had an alternating pattern. This was statistically significant to differentiate ET rest tremor from PD tremor [44]. Similar results were reported recently by Arabia, et al. They performed electrophysiological studies on 37 tremor predominant PD patients, 48 ET patients and 19 ET-PD patients (ET patients who developed PD). ET-PD patients showed a synchronous pattern of resting tremor in contrast to $P D$ patients. They also had an abnormal blink-reflex recovery cycle, an electrophysiological feature not seen in ET patients. It was concluded that these two electrophysiological parameters can be used to differentiate between ET, tremor predominant PD and ET-PD phenotypes [45]. These results need to be verified in larger cohorts.

\section{Essential Tremor and Imaging}

Advanced imaging techniques, including standard MRI, diffusion MRI and PET scan, have been studied to determine the structural substrate in ET patients. Most of these studies pointed towards cerebellum or the related networks as the likely anatomical structure implicated in the pathogenesis of ET [46]. Diffusion tensor imaging is an MRI technique that determines water diffusion (mean diffusivity, $M D$ ) and diffusion directionality (fractional anisotropy, FA). This technique is helpful to determine axonal injury or white matter disruption. Recently, Juttukonda MR, et al. conducted a study to assess white matter integrity in patients with ET and PD. They reported that FA values helped to differentiate ET from PD. Based on these values, white matter microstructural changes in ET localized to thalamus, visual related pathways and cerebellar peduncles. This was not observed in PD patients. These 
findings corroborated prior functional imaging studies and suggested that pathologic white matter changes involving the afferent and efferent pathways of the cerebellum occur in patients with ET as compared to PD. Understanding of these structural changes will not only help to identify pathogenic mechanisms underlying ET but also provide us with a biologic marker to distinguish between ET and PD [47].

\section{Mortality in ET}

Risk of mortality in ET patients was studied in a longitudinal, prospective study of 201 ET cases. They were compared with 465 age-matched controls. Risk of mortality was increased in ET subjects as compared to controls [48]. Louis, et al. conducted a longitudinal, prospective study of $141 \mathrm{ET}$ cases to determine predictors of mortality in ET patients. Older age, cognitive decline, depression, imbalance, and falls were associated with increased risk of mortality. Older age, cognitive decline and depression were independently associated with increased risk of mortality [49]. Despite its limitations, this study reinforced the notion that ET patients should be evaluated comprehensively, and regular monitoring of gait, cognition and mood should be done.

\section{Treatment of ET}

Medical management of Essential Tremor has not evolved much. Despite growing understanding of neuropathology, clinical features and non-motor symptoms, first line treatment of the syndrome remains the same. Primidone, propranolol and topiramate are the first line medications for ET. Botulinum toxin injections have been used for the treatment of ET for over 2 decades, but the use is still off-label. Two prior studies on use of botulinum toxin injection in essential tremor reported improvement in hand tremor. But the injections were given in fixed dose and fixed muscles [50,51]. Recently, Mittal, et al. conducted the first randomized, double-blind, placebo-controlled study with customized injection of botulinum toxin injection for ET patients. 80-120 units were injected into 8-14 hand and forearm muscles. These muscles were selected after careful clinical examination and these findings were confirmed via needle electromyography (EMG). Muscle selection for every patient was different based on tremor activity noted on examination and EMG. These patients were followed for 28 weeks and the treatment efficacy was evaluated by the FahnTolosa Marin tremor rating score and NIH genetic criteria for tremor severity at 4 and 8 weeks after each of the two sets of treatments. Statistically significant improvement in tremor score at 4 and 8 weeks after botulinum toxin injection was reported [52]. These findings merit further validation via larger study but assures that further improvement in injection technique will provide more options for management of ET patients.

Surgical management of Essential Tremor has evolved over last few years. Deep brain stimulation (DBS) of ViM nucleus of thalamus and thalamotomy are FDA approved surgical options for patients with ET [53]. DBS implants have been upgraded with time and implants by different vendors have been approved. This has broadened the options available for neurostimulation. With availability of rechargeable DBS batteries, patients don't have to undergo battery replacement frequently. Field of neurostimulation hold more promises in the future, as directional leads, adapative DBS and more refined platforms for programming become available [54].

In 2016, New England Journal of Medicine published results of a randomized trial of focused ultrasound thalamotomy for ET. 76 patients with refractory, severe ET were included. MRI guided, transcranial, unilateral focused ultrasound thalamotomy significantly reduced hand tremor at 3 months and the effect persisted till end of the 12-month study [55]. Comparison between DBS and ultrasound guided thalamotomy for ET patients is not available, but it is clear that latter is an irreversible procedure with no further modification possible. So far, in most of the practices, ablative procedures are reserved for patients who are either not willing to undergo intracranial surgery or those who are medically not fit for surgery and/or anesthesia. Nevertheless, it is a new procedure with its pros and cons and should be kept in mind when evaluating a refractory ET patient.

Essential Tremor remains a broad term and likely encompass a variety of tremor syndromes. We have developed our understanding of ET over last few decades but much needs to be explored and clarified. Recent updates have helped to answer some of the questions but have added few to the list that needs to be answered. For instance, how well the new classification scheme will be implemented in clinical practice and how is it going to impact ET research? Is age related tremor a distinct entity or perhaps ET plus? Are memory changes in ET indicative of a progressive neurodegenerative disorder? Is there a preclinical phase for ET? Do we need to develop new assessment scales for tremor that will include other neurological signs seen in ET plus? What will be the long-term effects of ultrasound guided thalamotomy in ET patients? Longitudinal studies of large cohorts of ET patients are much needed to validate some of the previous work.

\section{References}

1. Louis ED, Ferreira JJ (2010) How common is the most adult movement disorder? Update on the worldwide prevalence of essential tremor. Mov Disord 25: 534-541.

2. Chouinard S, Louis ED, Fahn S (1997) Agreement among movement disorder specialists on the clinical diagnosis of essential tremor. Mov Disord 12: 973-976.

3. Deuschl G, Bain P, Brin M (1998) Consensus statement of the Movement Disorder Society on Tremor. Ad Hoc Scientific Committee. Mov Disord 13: 2-23. 
4. Jankovic J (2002) Essential tremor: a heterogenous disorder. Mov Disord 17: 638-644.

5. Louis ED (2018) Essential tremor then and now: How views of the most common tremordiathesis have changed over time. Parkinsonism Relat Disord 46: S70-S74.

6. Elble RJ (2002) Essential tremor is a monosymptomatic disorder. Mov Disord 17: 633-637.

7. Benito-Leon J (2008) Essential Tremor: from a monosymptomatic disorder to a more complex entity. Neuroepidemiology 31: 191-192.

8. Bhatia KP, Bain P, Bajaj N, Elble RJ, Hallet M, et al. (2018) Consensus statement on the classification of tremors. From the task force on tremor of the International Parkinson and Movement Disorder Society. Mov Disord 33: 75-87.

9. Gulcher JR, Jonsson P, Kong A, Kristjansson K, Frigge ML, et al. (1997) Mapping of a familial essential tremor gene, FET1, to chromosome3q13. Nat Genet 17: 84-87.

10. Higgins JJ, Pho LT, Nee LE (1997) A gene (ETM) for essential tremor maps to chromosome 2p22-p25. Mov Disord 12: 859-864.

11. Shatunov A, Sambuughin N, Jankovic J, Elble A, Lee HS, et al. (2006) Genomewide scans in North American families reveal genetic linkage of essential tremor to a region on chromosome 6p23. Brain 129: 2318-2331.

12. Louis ED, Ford B, Frucht S, Ottman R (2001) Mild tremor in relatives of patients with essential tremor: what does this tell us about the penetrance of the disease? Arch Neurol 58: 1584-1589.

13. Louis ED, Factor-Litvak P, Liu X, Vonsattel JP, Galecki $M$, et al. (2013) Elevated brain harmane (1-methyl-9Hpyrido[3,4-b]indole) inessential tremor cases vs. controls. Neurotoxicology 38: 131-135.

14. Dogu O, Louis ED, Tamer L, Unal O, Yilmaz A, et al. (2007) Elevated blood lead concentrations in essential tremor: a case-control study in Mersin, Turkey. Environ Health Perspect 115: 1564-1568.

15. Louis ED, Benito-Leon J, Bermejo-Pareja F (2009) Population-based study of baseline ethanol consumption and risk of incident essential tremor. J Neurol Neurosurg Psychiatry 80: 494-497.

16. ED Louis (2014) Re-thinking the biology of essential tremor: from models to morphology. Parkinsonism Relat Disord 20: S88-S93.

17. Louis ED, Yi H, Erickson-Davis C, Vonsattel JP, Faust PL (2009) Structural study of Purkinje call axonal torpedoes in essential tremor. Neurosci Lett 450: 287-291.

18. Lin CY, Louis ED, Faust PL, Koeppen AH, Vonsattel JP, et al. (2014) Abnormal climbing fibre-Purkinje cell synaptic connections in essential tremor cerebellum. Brain 137: 3149-3159.

19. Louis ED, Faust PL, Vonsattel JP, Honig LS, Rajput A, et al. (2007) Neuropathological changes in essential tremor: 33 cases compared with 21 controls. Brain 130: 3297-3307.

20. Louis ED, Honig LS, Vonsattel JP, Maraganore DM, Borden S, et al. (2005) Essential Tremor associated with focal nonnigral Lewy bodies: a clinicopathological study. Arch Neurol 62: 1004-1007.

21. Louis ED, Mazzoni P, Ma KJ, Moskowitz CB, Lawton A, et al. (2012) Essential tremor with ubiquitinated intranuclear inclusions and cerebellar degeneration. Clin Neuropathol 31: $119-126$.
22. Deuschl G, Petersen I, Lorenz D, Christensen K (2015) Tremor in the elderly: essential and aging-related tremor. Mov Disord 30: 1327-1334.

23. Hopfner F, Ahlf A, Lorenz D, Kelbe S, Zeuner G, et al. (2016) Early- and late-onset essential tremor patients represent clinically distinct subgroups. Mov Disord 31: 1560-1566.

24. Kuo SH, Wang J, Tate WJ, Pan MK, Kelly GC, et al. (2017) Cerebellar pathology in early onset and late onset essential tremor. Cerebellum 16: 473-482.

25. Sinoff G, Badarny S (2014) Mild cognitive impairment, dementia, and affective disorders in essential tremor: a prospective study. Tremor Other Hyperkinet Mov 4: 227.

26. Benito-Leon J, Louis ED, Bermejo-Pareja F (2006) Elderlyonset essential tremor is associated with dementia. Neurology 66: 1500-1505.

27. Lombardi WJ, Woolston DJ, Roberts JW, Gross RE (2001) Cognitive deficits in patients with essential tremor. Neurology 57: 785-790.

28. Benito-Leon J, Louis ED, Sanchez-Ferro E, Bermejo-Pareja F (2013) Rate of cognitive decline during the pre-motor phase of essential tremor: a prospective study. Neurology 81: 8160-8166.

29. Lacrtiz LH, Dewey r Jr, Giller C, Cullum CM (2002) Cognitive functioning in individuals with "benign" essential tremor. J Int Neuropsychol Soc 8: 125-129.

30. Troster Al, Woods SP, Fields JA, Lyons KE, Pahwa R, et al. (2002) Neuropsychological deficits in essential tremor: an expression of cerebello-thalamo-cortical pathophysiology? Eur J Neurol 9: 143-151.

31. Cersonsky TEK, Morgan S, Kellner S, Robakis D, Liu X, et al. (2018) Evaluating mild cognitive impairment in essential tremor: how many and which neuropsychological tests? J Int Neuropsychol Soc 24: 1084-1098.

32. Park IS, Oh YS, Lee KS, Yang DW, Song IU, et al. (2015) Subtype of mild cognitive impairment in elderly patients with essential tremor. Alzheimer Dis Assoc Disord 29: 141-145.

33. Collins K, Rohl B, Morgan S, Huey ED, Louis ED, et al. (2017) Mild cognitive impairment subtypes in a cohort of elderly essential tremor cases. J Int Neuropsychol Soc 23: 390-399.

34. Louis ED, Rios E, Rao AK (2010) Tandem gait performance in essential tremor: clinical correlates and association with midline tremors. Mov Disord 25: 1633-1638.

35. Kronenbuerger $M$, Konczack J, Ziegler W, Buderath $P$, Frank B, et al. (2009) Balance and motor speech impairment in essential tremor. Cerebellum 8: 389-398.

36. Roemmich RT, Zeilman PR, Vaillancourt DE, Okun MS, Hass CJ (2013) Gait variability magnitude but not structure is altered in essential tremor. J Biomech 46: 2682-2687.

37. Louis ED, Rao AK, Gerbin M (2012) Functional correlates of gaitand balance difficulty in essential tremor: balance confidence, near misses and falls. Gait Posture 35: 43-47.

38. Rao A, Gillman A, Louis ED (2011) Quantitative gait analysis in essential tremor reveals impairments that are maintained into advanced age. Gait Posture 34: 65-70.

39. Louis ED, Meyers JH, Cristal AD, Hickman R, Factor-Litvak $P$ (2018) Unaffected first-degree relatives of essential tremor cases have more imbalance than age-matched control subjects. Parkinsonism Relat Disord 52: 24-29.

40. Fabbrini G, Berardeli I, Falla M, Moretti G, Pasquini M, et al. (2012) Psychiatric disorders in patients with essential tremor. Parkinsonism Relat Disord 18: 971-973. 
41. Louis ED, Benito-Leon J, Bermejo-Pareja F (2007) Selfreported depression and anti-depressant medication use in essential tremor: cross-sectional and prospective analyses in a population based study. Eur J Neurol 14: 1138-1146.

42. Li ZW, Xie MJ, Tian DS, Li JJ, Zhang JP, et al. (2011) Characteristics of depressive symptoms in essential tremor. J Clin Neurosci 18: 52-56.

43. Aslam S, Zhang N, Adler $\mathrm{CH}$, Caviness JN, DriverDunckley E, et al. (2017) Essential tremor and depression. Mov Disord Clin Pract 4: 836-842.

44. Nistico R, Pirritano D, Salsone M, Novellino F, Del Giudice $F$, et al. (2011) Synchronous pattern distinguishes resting tremor associated with essential tremor from rest tremor of Parkinson's disease. Parkinsonism and Related disorder 17: 30-33.

45. Arabia G, Lupo A, Manfredini LI, Vescio B, Nistico R, et al. (2018) Clinical, electrophysiological, and imaging study in essential tremor-Parkinson's disease syndrome. Parkinsonism and Related Disorders 56: 20-26.

46. Klaming R, Annese J (2014) Functional anatomy of essential tremor: lessons from neuroimaging. AJNR Am J Neuroradiol 35: 1450-1457.

47. Juttukonda MR, Franco G, Englot DJ, Lin YC, Petersen KJ, et al. (2019) White matter differences between essential tremor and Parkinson disease. Neurology 92: e30-e39.

48. Louis ED, Benito-Leon J, Ottoman R, Bermejo-Pareja F (2007) Neurological disorders in Central Spain (NEDICES) study group. A population based study of mortality in essential tremor. Neurology 69: 1982-1989.
49. Zubair A, Cersonsky TEK, Kellner S, Huey ED, Cosentino $S$, et al. (2018) What predicts mortality in essential tremor? A prospective, longitudinal study of elders. Front Neurol 9: 1077.

50. Brin MF, Lyons KE, Doucette J, Adler CH, Caviness JN, et al. (2001) A randomized, double masked, controlled trial of botulinum toxin type $A$ in essential hand tremor. Neurology 56: 1523-1528.

51. Jankovic J, Schwartz K, Clemence W, Aswad A, Mordaunt J (1996) A randomized, double-blind, placebo-controlled study to evaluate botulinum toxin type $A$ in essential hand tremor. Mov Disord 11: 250-256.

52. Mittal SO, Machado D, Richardson D, Dubey D, Jabbari B (2018) Botulinum toxin in essential hand tremor- a randomized double-blind placebo-controlled study with customized injection approach. Parkinsonism Relat Disord 56: 65-69.

53. Zesiewicz TA, Elble R, Louis ED, Hauser RA, Sullivan KL, et al. (2005) Practice parameter: therapies for essential tremor: report of the quality standards subcommittee of the American Academy of Neurology. Neurology 64: 20082020.

54. Neumann WJ, Turner RS, Blankertz B, Mitchell T, Kuhn AA, et al. (2019) Toward electrophysiology-based intelligent adaptive deep brain stimulation for movement disorders. Neurotherapeutics 16: 105-118.

55. Elias WJ, Lipsman N, Ondo WG, Ghanouni P, Kim YG, et al. (2016) A randomized trial of focused ultrasound thalamotomy for essential tremor. N Engl J Med 375: 730739. 